\title{
DISTRIBUCIÓN DE ESCAÑOS PARA DIPUTADOS COSTARRICENSES
}

\section{DISTRIBUTION OF PARLAMENTARY SEATS FOR COSTARRICAN LEGISLATORS}

\section{Luis Diego Brenes Villalobos*}

RESUMEN

El artículo describe la forma en cómo se distribuyen los escaños de diputados en Costa Rica, mediante un listado de 10 pasos que refieren a todos los elementos clave del sistema electoral: circunscripción, magnitud, forma de la candidatura, procedimiento de votación y fórmula electoral.

PALABRAS CLAVE: COSTA RICA * SISTEMAS ELECTORALES * PARLAMENTO * ELECCIONES

ABSTRACT

The article describes how the parliamentary seats are distributed in Costa Rica, through a list of 10 steps thatare related to all the key elements of the electoral system: district, magnitude, type of nominations, voting procedureand electoral formula.

KEYWORDS: COSTA RICA * ELECTORAL SYSTEMS* PARLAMENT * ELECTIONS

Facultad de Derecho de la Universidad de Costa Rica (UCR), Universidad Estatal a Distancia de Costa Rica (UNED) y Tribunal Supremo de Elecciones de Costa Rica (TSE).

dbrenes@tse.go.cr 


\section{INTRODUCCIÓN}

La elección de los 57 diputados que integran la Asamblea Legislativa en Costa Rica, se realiza por provincias mediante listas "cerradas $y$ bloqueadas" con votación única y tomando como fórmula matemática para su distribución, las cifras cociente y residuales. Adicionalmente, el método de adjudicación de plazas fija una barrera electoral — como umbral mínimo para entrar en el reparto - determinada por la cifra subcociente (Brenes, 2006, 2007 y 2014).

La lectura puntual de los artículos 201 a 205 del Código Electoral, dispone cómo se debe realizar esa adjudicación de plazas; sin embrgo, el presente artículo pretende una descripción, en 10 pasos, de la forma en que se debe realizar esa distribución. El objetivo pasa por una suerte de manual de referencia para concretar esa distribución.

La descripción y "receta" de 10 pasos que se expone, procura un lenguaje sencillo con explicación de todos los elementos electorales involucrados. Asimismo, en aras de esa simplicidad y mejor comprensión, se omite referencia a tecnicismos, abuso de citas legales $y$ doctrinarias ${ }^{1}$.

Como ejemplo para la explicación de la distribución, se utiliza un caso real de las

1 Para un estudio más detallado, incluso de carácter comparado con otras fórmulas electorales y de naturaleza diacrónica (1953-2010), puede verse la investigación del mismo autor: "Cambios sin reforma: sistema de partidos y barrera electoral en Costa Rica (1953-2010)". Revista Centroamericana de Ciencias Sociales 1 (VIII). Julio 2011: 5-35. elecciones del año 2006. El caso refiere a la distribución de escaños de la provincia de Limón, siguiendo al efecto la resolución del Tribunal Supremo de Elecciones (TSE) nro. 1137-E-2006 del 22 de marzo de 2006, en la cual se constituye la Declaratoria de Elección de Diputados a la Asamblea Legislativa para el período 2006-2010².

Los 10 pasos a seguir son: 1) ordenar la votación partidaria, 2) eliminación de votos nulos y votos en blanco, 3) cálculo del dividendo, 4) delimitación del divisor, 5) cálculo del cociente, 6) cálculo del subcociente, 7) distribución por cociente, 8) cálculo de cifras residuales, 9) distribución por resto mayor y 10) declaratoria de elección.

\section{DESCRIPCIÓN EN 10 PASOS}

\section{ORDENAR LA VOTACIÓN PARTIDARIA}

El primer paso consiste en ordenar los resultados que se reciben después de una votación. Aunque este paso no es estrictamente necesario, se recomienda porque facilita la lectura y posterior distribución de plazas (también llamados y conocidos como escaños o curules). Simplemente debe ordenarse la votación que reciben todos los partidos políticos en forma decreciente: el más votado de primero en la lista.

2 Las resoluciones y normativa mencionadas a lo largo del presente artículo pueden ser consultadas en el sitio web: www.tse.go.cr. 


\section{CUADRO 1 \\ VOTACIÓN EN ORDEN DECRECIENTE. ELECCIÓN LEGISLATIVA PROVINCIA DE LIMÓN, 2006}

\begin{tabular}{lcc}
\hline PARTIDO POLÍTICO & SIGLA & VOTOS VÁLIDOS \\
\hline Partido Liberación Nacional & PLN & 38669 \\
Partido Acción Ciudadana & PAC & 18223 \\
Partido Unidad Social Cristiana & PUSC & 12890 \\
Movimiento Libertario & ML & 12264 \\
Partido Renovación Costarricense & PRC & 7545 \\
Partido Unión Nacional & PUN & 3970 \\
Partido Unión Para el Cambio & PUPC & 3240 \\
Fuerza Democrática & FD & 1632 \\
Movimiento de Trabajadores y Campesinos & MTC & 1507 \\
Partido Patria Primero & PP & 1414 \\
Coalición Izquierda Unida & CIU & 1046 \\
Partido Integración Nacional & PIN & 861 \\
Partido Unión Patriótica & PUP & 724 \\
Partido Alianza Democrática Nacionalista & ADN & 665 \\
Votos nulos & & 3962 \\
Votos en blanco & & 1626 \\
\hline
\end{tabular}

Fuente: Elaboración propia.

\section{ELIMINACIÓN DE VOTOS NULOS Y VOTOS EN BLANCO}

Los votos nulos y los votos en blanco no cuentan ni en la elección de Presidente ni en la de diputados, tampoco en las elecciones municipales. Estos votos no se suman al candidato más votado ni tampoco se ponderen para el cálculo de umbrales o barreras electorales.

Si bien, los votos nulos y en blanco tienen un valor políticamente hablando, jurídicamente no tienen peso alguno en el resultado final. Los votos nulos $y$ en blanco se reportan entonces como una cifra y estadística más del proceso ${ }^{3}$.

$3 \quad$ Los votos nulos y en blanco no se tienen en el sistema costarricense como votos válidamente

\section{CÁLCULO DEL DIVIDENDO}

La división a la que se procederá requiere determinar sus elementos. El primero de ellos, la cifra que va arriba de la división (dividendo), la constituye la sumatoria de las votaciones logradas por todos los partidos que participaron en una provincia. En este cálculo no deben sumarse los votos nulos y en blanco, justamente eliminados en el paso anterior.

emitidos. En tal sentido pueden consultarse las resoluciones del TSE nro. 2587-E-2001 de las 14 horas del 29 de noviembre de 2001 y nro. 2618E-2005 de las 12:30 horas del 7 de noviembre de 2005 (Brenes, 2007:130). 
CUADRO 2

CÁLCULO DEL DIVIDENDO. ELECCIÓN

LEGISLATIVA

PROVINCIA DE LIMÓN, 2006

\begin{tabular}{cc}
\hline SIGLA & VOTOS VÁLIDOS \\
\hline PLN & 38669 \\
PAC & 18223 \\
PUSC & 12890 \\
ML & 12264 \\
PRC & 7545 \\
PUN & 3970 \\
PUPC & 3240 \\
FD & 1632 \\
MTC & 1507 \\
PP & 1414 \\
CIU & 1046 \\
PIN & 861 \\
PUP & 724 \\
ADN & 665 \\
\hline
\end{tabular}

Total de votos (sumatoria)

Fuente: Elaboración propia.

\section{DELIMITACIÓN DEL DIVISOR}

El divisor, parte de abajo de la división, está representado por el número de escaños a distribuir en una provincia. La propia Constitución Política (artículo 106) fija en 57 el número de diputados para todo el país y establece que cada vez que se realice un censo general de población, el TSE asignará a las provincias, las diputaciones respectivas en proporción a la población de cada una de ellas.

El decreto de convocatoria a elecciones - que en forma pública $y$ acto solemne se celebra 4 meses antes de una elección- es donde se expresa el número de diputados que corresponde a cada provincia. El Tse realiza un cálculo de estricta proporcionalidad para determinar el número de diputados por provincia y así las reporta. Consecuentemente, el divisor no es necesario calcularlo, es un número dado por el propio TSE.

Para las elecciones 2014, los divisores por provincia se fijaron en: San José (19), Alajuela (11), Cartago (7), Heredia (6), Guanacaste (4),
Puntarenas (5) y Limón (5). Esas cifras, se conocen como la "magnitud" de cada circunscripción electoral. Ahora bien, en el caso que se utiliza de ejemplo en esta descripción, la provincia de Limón para las elecciones del 2006 y conforme al último censo general de población —en aquel momento era el del año 2001- se escogió por igual un total de cinco diputados.

El número cinco constituirá entonces el factor "divisor" en la división matemática requerida para el próximo cálculo de la cifra cociente.

\section{CÁLCULO DEL COCIENTE}

Una división es el resultado de dividir el dividendo entre el divisor. A ese resultado también se le denomina cifra cociente o cuota. En el ejemplo, el cociente es el resultado de dividir la sumatoria de la votación de todos los partidos en una provincia (paso 3) entre el número de escaños a repartir en esa provincia (paso 4$)^{4}$.

Cociente $=\underline{\text { Total de votos válidos (en cada provincia) }}$

Número de plazas

CUADRO 3

CÁLCULO DEL COCIENTE. ELECCIÓN

LEGISLATIVA

PROVINCIA DE LIMÓN, 2006

\begin{tabular}{llll}
\hline FÓRMULA & TOTAL DE & NÚMERO DE & CIFRA \\
MATEMÁTICA & VOTOS & PLAZAS & COCIENTE \\
& $\begin{array}{l}\text { VÁLIDOS } \\
\text { (DIVIDENDO) }\end{array}$ & & \\
\hline División & 104650 & 5 & 20930 \\
\hline
\end{tabular}

Fuente: Elaboración propia.

\section{CÁlCULO DEL SUBCOCIENTE}

El subcociente es de una aritmética aún más fácil: es simplemente la mitad del cociente; es decir, el subcociente es el resultado de dividir la cifra cociente (paso 5) entre dos ${ }^{5}$.

$4 \quad$ Según el párrafo primero del artículo 203 del Código Electoral: "cociente es la cifra que se obtiene dividiendo el total de votos válidos emitidos para determinada elección, entre el número de plazas a llenar mediante dicha elección".

$5 \quad$ Conforme al párrafo segundo del artículo 203 del Código Electoral: "subcociente es el total de 


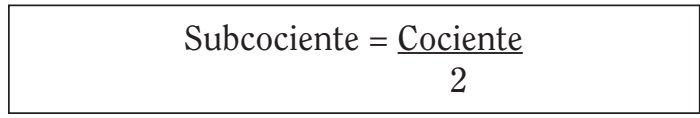

CUADRO 4

CÁLCULO DEL SUBCOCIENTE. ELECCIÓN LEGISLATIVA

PROVINCIA DE LIMÓN, 2006

\begin{tabular}{cccc}
\hline $\begin{array}{c}\text { FÓRMULA } \\
\text { MATEMÁTICA }\end{array}$ & $\begin{array}{c}\text { COCIENTE } \\
\text { (DIVIDENDO) }\end{array}$ & $\begin{array}{c}\text { MITAD } \\
\text { (DIVISOR) }\end{array}$ & $\begin{array}{c}\text { CIFRA SUB } \\
\text { COCIENTE }\end{array}$ \\
\hline $1 / 2$ del Cociente & 20930 & 2 & 10465 \\
\hline
\end{tabular}

Fuente: Elaboración propia.

La clave de la cifra subcociente radica en comprender que esta es una barrera electoral, de manera que los partidos que no alcanzan esa cifra quedan excluidos del reparto de plazas. El subcociente entonces, como umbral mínimo de participación, elimina partidos y verifica entre cuales se formalizará el reparto.

De esta manera, en el ejemplo que nos ocupa de la provincia de Limón, en tanto solo cuatro agrupaciones, del total de 14 que participaron, lograron superar la barrera del subcociente, únicamente entre estas cuatro agrupaciones se verifica el cálculo para la distribución de escaños. Dicho de otra manera, las otras 10 agrupaciones políticas no entran en consideración en el reparto de plazas dado que sus votaciones no superaron el umbral que constituye el subcociente.

votos válidos emitidos a favor de un partido que, sin alcanzar la cifra cociente, alcanza o supera el cincuenta por ciento (50\%), de esta".
CUADRO 5

ELIMINACIÓN DE PARTIDOS POR SUBCOCIENTE ELECCIÓN LEGISLATIVA PROVINCIA DE LIMÓN, 2006

\begin{tabular}{ll}
\hline SIGLA & VOTOS VÁLIDOS \\
\hline PLN & 38669 \\
PAC & 18223 \\
PUSC & 12890 \\
ML & 12264 \\
Barrera del subcociente & 10465 \\
PRE & 7545 \\
PUN & 3970 \\
PUPC & 3240 \\
FD & 1632 \\
MTC & 1507 \\
PP & 1414 \\
CIU & 1046 \\
PIN & 861 \\
PUP & 724 \\
ADA & 665 \\
\hline
\end{tabular}

Fuente: Elaboración propia.

Nota: Se destaca donde queda ubicada la barrera del subcociente y la consecuente eliminación de las agrupaciones que no superan esa barrera.

\section{DISTRIBUCIÓN POR COCIENTE}

Se regresa en este punto a la cifra cociente (paso 5). Los partidos que en sus votaciones iniciales, individualmente consideradas, alcanzan o superan la cifra cociente, tienen derecho 
a escaños ${ }^{6}$. Dado que un cociente equivale a una curul, tendrán derecho a tantos escaños como cocientes logren. En el ejemplo de referencia, la cifra cociente que equivale a un escaño es 20930.

Eventualmente, todas las plazas a distribuir en una provincia pueden repartirse entre los cocientes logrados. Pero, de no presentarse esta situación, escenario que es muy usual - tal cual sucede en el presente ejemplo-, es necesario recurrir a las cifras residuales para las restantes plazas pendientes de asignación.

Una manera de verificar cuántas veces "entra" la cifra cociente en la votación partidaria es dividiendo el total de votos válidos entre la cifra cociente. Evidentemente, ese resultado debe redondearse al número entero inferior.

\section{CUADRO 6 \\ DISTRIBUCIÓN POR COCIENTE. ELECCIÓN LEGISLATIVA PROVINCIA DE LIMÓN, 2006}

\begin{tabular}{|c|c|c|c|c|}
\hline $\begin{array}{l}\text { PARTIDO } \\
\text { POLÍTICO }\end{array}$ & $\begin{array}{l}\text { VOTOS } \\
\text { VÁLIDOS }\end{array}$ & $\begin{array}{l}\text { CIFRA } \\
\text { COCIENTE }\end{array}$ & $\begin{array}{l}\text { CÁLCULO DE CIFRA COCIENTE } \\
\text { EN VOTACIÓN }\end{array}$ & $\begin{array}{l}\text { NÚMERO DE ESCAÑOS POR } \\
\text { CIFRA RESIDUAL }\end{array}$ \\
\hline PLN & 38669 & 20930 & 1,84 & 1 \\
\hline PAC & 18223 & - & - & 0 \\
\hline PUSC & 12890 & - & - & 0 \\
\hline $\mathrm{ML}$ & 12264 & - & - & 0 \\
\hline
\end{tabular}

Fuente: Elaboración propia.

\section{CÁLCULO DE CIFRAS RESIDUALES}

Las cifras residuales se calculan de dos maneras: por un lado entre los partidos que superaron la cifra cociente (color gris oscuro en cuadros 7 y 8) y por otro, entre los partidos que si bien no alcanzaron la cifra cociente, sí superaron la barrera del subcociente.

Entre los partidos que alcanzaron la cifra cociente y que por esta se les asignó uno o varios escaños, la cifra residual es la resta a la votación inicial de ese partido de la cifra cociente, cuantas veces se haya alcanzado. En el ejemplo, este es el caso que corresponde únicamente al pLN.

Por otra parte, a los partidos que superaron la cifra subcociente pero no alcanzaron la cifra cociente ( agrupaciones que no quedaron excluidas por la barrera pero no entraron en el primer reparto por cociente, se les tienen sus votaciones iniciales como cifras residuales para entonces ser considerados en el segundo reparto.

$6 \quad$ Al efecto, el numeral 205 del Código Electoral establece: "en los casos de elección por cociente y subcociente, a cada partido que haya concurrido a la votación se le declarará elegido(a) en el orden de su colocación en la papeleta, por el electorado de que

se trate, tantos candidatos(as) como cocientes haya logrado. Primero se hará la declaratoria de elección del partido que mayor número de votos obtuvo en el circuito electoral de que se trate; se continuará en el orden decreciente de los partidos". 


\section{CUADRO 7 \\ CÁLCULO DE CIFRAS RESIDUALES. ELECCIÓN LEGISLATIVA PROVINCIA DE LIMÓN, 2006}

\begin{tabular}{ccccc}
\hline PARTIDO POLÍTICO & VOTOS VÁLIDOS & CIFRA COCIENTE & $\begin{array}{c}\text { NÚMERO DE ESCAÑOS POR } \\
\text { CIFRA COCIENTE }\end{array}$ & CIFRA RESIDUAL \\
\hline PLN & 38669 & 20930 & 1 & -17739 \\
PAC & 18223 & - & 0 & -18223 \\
PUSC & 12890 & - & 0 & -12890 \\
ML & 12264 & - & 0 & -12264 \\
\hline
\end{tabular}

Fuente: Elaboración propia.

Nota: $\quad$ El carácter de signo negativo (-) de previo a las cifras residuales es para resaltar ese carácter de resta, no por un eventual valor negativo (menor a cero) de la cifra.

\section{DISTRIBUCIÓN POR RESTO MAYOR}

Para las plazas pendientes de distribuir en una provincia, se recurre entonces a las mayores cifras residuales. En el ejemplo, en tanto solo un escaño se asignó por cociente y dado que son 5 las plazas a repartir, debe distribuirse por cifras residuales un total de 4 plazas. Para esa nueva distribución o segundo reparto, la asignación se hace de mayor a menor entre las cifras residuales.

\section{CUADRO 8 \\ DISTRIBUCIÓN POR RESTO MAYOR. ELECCIÓN LEGISLATIVA. PROVINCIA DE LIMÓN, 2006}

\begin{tabular}{|c|c|c|c|c|c|}
\hline $\begin{array}{l}\text { PARTIDO } \\
\text { POLÍTICO }\end{array}$ & $\begin{array}{l}\text { VOTOS } \\
\text { VÁLIDOS }\end{array}$ & $\begin{array}{c}\text { CIFRA } \\
\text { COCIENTE }\end{array}$ & $\begin{array}{l}\text { NÚMERO DE ESCAÑOS } \\
\text { POR CIFRA COCIENTE }\end{array}$ & $\begin{array}{c}\text { CIFRA } \\
\text { RESIDUAL }\end{array}$ & $\begin{array}{l}\text { NÚMERO DE ESCAÑOS } \\
\text { POR CIFRA RESIDUAL }\end{array}$ \\
\hline PLN & 38669 & 20930 & 1 & -17739 & 1 \\
\hline PAC & 18223 & - & 0 & -18223 & 1 \\
\hline PUSC & 12890 & - & 0 & -12890 & 1 \\
\hline ML & 12264 & - & 0 & -12264 & 1 \\
\hline
\end{tabular}

Fuente: Elaboración propia.

Nota: El carácter de signo negativo (-) de previo a las cifras residuales es para resaltar ese carácter de resta o residuo, no por un eventual valor negativo (menor a cero) de la cifra.

Si hubiese más plazas a distribuir que cifras residuales, o lo que es lo mismo, si asignadas plazas a las cifras residuales aún quedan escaños pendientes de distribuir (tercer reparto que no sucede en el ejemplo de referencia), la normativa ordena repetir este mismo sistema de asignación, de manera que se vuelven a revisar las cifras residuales de mayor a menor, $y$ se asignan entre estas?

$7 \quad$ La normativa al efecto, párrafos segundo y tercero del artículo 205 del Código Electoral indican:"si

quedan plazas sin llenar por el sistema de cociente, la distribución de estas se hará a favor de los partidos en el orden decreciente de la cifra residual de su votación, pero incluyendo, también, los partidos que apenas alcanzaron subcociente, como si su votación total fuera cifra residual. Si aún quedan plazas sin llenar, se repetirá la operación que se expresa en el aparte anterior". 
10. DECLARATORIA DE ELECCIÓN.

Realizada la adjudicación de todas las plazas, lo único pendiente es la respectiva declaratoria de elección. Para el caso de los diputados, importa anotar que el Tse cuenta con 60 días, posteriores a la fecha de celebración de la elección, para finalizar con el escrutinio.

\section{DECLARATORIA DE ELECCIÓN. ELECCIÓN LEGISLATIVA PROVINCIA DE LIMÓN, 2006}

\begin{tabular}{ccccccc}
\hline $\begin{array}{c}\text { PARTIDO } \\
\text { POLÍTICO }\end{array}$ & $\begin{array}{c}\text { VOTOS } \\
\text { VÁLIDOS }\end{array}$ & $\begin{array}{c}\text { CIFRA } \\
\text { COCIENTE }\end{array}$ & $\begin{array}{c}\text { NÚMERO DE } \\
\text { ESCAÑOS POR } \\
\text { CIFRA COCIENTE }\end{array}$ & $\begin{array}{c}\text { CIFRA } \\
\text { RESIDUAL }\end{array}$ & $\begin{array}{c}\text { NÚMERO DE } \\
\text { ESCANONOS POR } \\
\text { CIFRA RESIDUAL }\end{array}$ & $\begin{array}{c}\text { TOTAL DE } \\
\text { ESCANOS POR } \\
\text { PARTIDO }\end{array}$ \\
\hline PLN & 38669 & 20930 & $1(1)$ & $-17739(3)$ & 1 & 2 \\
PAC & 18223 & - & 0 & $-18223(2)$ & 1 & 1 \\
PUSC & 12890 & - & 0 & $-12890(4)$ & 1 & 1 \\
ML & 12264 & - & 0 & $-12264(5)$ & 1 & 1 \\
\hline
\end{tabular}

Fuente: Elaboración propia.

Nota: $\quad$ El carácter de signo negativo (-) de previo a las cifras residuales es para resaltar ese carácter de resta o residuo, no por un eventual valor negativo (menor a cero) de la cifra. Asimismo, los números anotados entre paréntesis refieren al orden en que se asignaron las plazas, particularmente importante al momento de considerar las cifras residuales.

\section{CONCLUSIÓN}

El sistema electoral es la fórmula de cómputo para convertir votos en escaños (Nohlen, 2000); es una técnica que a la luz del principio de representación, materializa votos en poder público.

Como se ha mencionado en otras oportunidades, la elección de diputados en Costa Rica se rige por un sistema proporcional. A diferencia de los sistemas mayoritarios, donde quien termina en primer lugar arrasa con todas las plazas en disputa, los sistemas proporcionales benefician una distribución de plazas más representativa, equitativa e incluso más justa, en tanto respeta en mayor medida la proporcionalidad de la votación que cada partido logra.

Asimismo, es relevante considerar que los sistemas electorales del mundo se rigen por una diversidad de fórmulas matemáticas, todas con variaciones en la proporcionalidad de sus resultados. No hay una única receta o modelo perfecto; de hecho, ese escenario variopinto mundial, incluso dentro de las modalidades estrictamente proporcionales, varía de mayor a menor proporcionalidad. Adicionalmente, no pueden dejarse de lado la totalidad de los elementos que constituyen al sistema electoral, de suerte que el tipo de circunscripción, la magnitud de esta (número de plazas a escoger en una circunscripción que siempre referirá a enteros - no a fracciones-) y la participación que finalmente se manifiesta en una determinada elección, constituyen una serie de variables que en su combinación pueden llevar a sobre o subrepresentación.

De momento el propósito del artículo es mostrar en forma sencilla cómo se formaliza esa distribución de escaños de diputados en Costa Rica, aunque evidentemente hay mucho más que decir y profundizar cuando a la luz de los principios de la representación y la proporcionalidad se encaran los efectos matemáticos $y$ alcances reales de cada uno de los elementos que integran el sistema electoral. 
BIBLIOGRAFÍA

LIBROS

Cox, Gary W. La coordinación estratégica de los sistemas electorales del mundo. España: Editorial Gedisa, 2004.

LeDuc, Lawrence; Niemi, Richard G. y Norris, Pippa (eds.). Comparing democracies 2. New challenges in the study of elections and voting. Reino Unido: Sage Publications, 2002.

García Soriano, María Vicenta. Elementos de derecho electoral. España: Editorial Tirant Lo Blanch, 1999.

Lijphart, Arend. Electoral systems and party systems: a study of twenty-seven democracies, 1945-1990. Reino Unido: Oxford University Press, 1994.

Nohlen, Dieter. "Sistemas electorales". Diccionario Electoral, Tomo II. Costa Rica. Instituto Interamericano de Derechos Humanos (IIDH)-Centro de Asesoría y Promoción Electoral (CAPEL), 2000: 1157-1149.

Pedicone de Valls, María Gilda. Derecho electoral. Argentina: Ediciones La Rocca, 2001.

Reynolds, Andrew; Reilly, Ben y Ellis, Andrew (eds.). Electoral system design: the new international IDEA handbook. Suecia: International Institute for Democracy and Electoral Assistance, 2005.

Vallès Casadevall, Joseph M. y Bosch Guardella, Agustí. Sistemas electorales y gobierno representativo. España: Editorial Ariel, 1997.

PUBLICACIONES PERIÓDICAS

Alfaro Redondo, Ronald. "Ingeniería electoral en Costa Rica: proporcionalidad $y$ representatividad ¿por dónde empezar?”. Revista Parlamentaria 10. Costa Rica. Asamblea Legislativa, agosto 2002: 231-258.

Brenes Villalobos, Luis Diego. "Matemáticas electorales". Foro de Opinión, La Nación. San José, Costa Rica. 17 de abril de 2006: 40A.

Brenes Villalobos, Luis Diego. "Matemáticas Electorales: distribución de escaños en elecciones diputadiles costarricenses del 2006 (análisis comparativo)". Revista de Derecho Electoral 3. Costa Rica. Tribunal Supremo de Elecciones, enerojunio 2007: 127-146.

Brenes Villalobos, Luis Diego. "Elecciones 2010: transformaciones inconclusas del sistema de partidos en Costa Rica". Revista de Derecho Electoral 10. Costa Rica. Tribunal Supremo de Elecciones, julio-diciembre 2010: 79-92.

Brenes Villalobos, Luis Diego. "Cambios sin reforma: sistema de partidos y barrera electoral en Costa Rica (1953-2010)". Revista Centroamericana de Ciencias Sociales1 (8). Costa Rica. Facultad Latinoamericana de Ciencias SocialesFLACSO, julio 2011: 3-35.

Brenes Villalobos, Luis Diego. "Elección de diputados". Foro de Opinión, Semanario Universidad. San José, Costa Rica. 12 de febrero de 2014: 23.

\section{TEXTOS ELECTRÓNICOS}

Tribunal Supremo de Elecciones (TSE). Declaratorias definitivas de elección. Costa Rica. 2014. En: <http://www.tse. go.cr/declaratorias.htm > [consultado el 26 de marzo de 2014].

Tribunal Supremo de Elecciones (TSE). Resoluciones electorales. Costa Rica. 2014. En: <http://www.tse.go.cr/ jurisprudencia.htm $>$ [consultado el 26 de marzo de 2014].

Código Electoral de la República de Costa Rica. Costa Rica. 2014. En: <http://www.tse. go.cr/pdf/normativa/codigoelectoral.pdf> [consultado el 28 de marzo de 2014].

Constitución Política de la República de Costa Rica. Costa Rica. 2014. En: <http://www. tse.go.cr/pdf/normativa/constitucion.pdf> [consultado el 28 de marzo de 2014].

Fecha de ingreso: 21/04/2014 Fecha de aprobación: 17/07/2014 
